identify the antimicrobial agents involved in bacterial resistance.

Methods A cross-sectional study, with data obtained retrospectively through medical records review. We analysed 140 patients whose women's endocervical samples and men's urine tested positive for $\mathrm{MH}$ and UU. The test used for the diagnosis and evaluation of antimicrobial resistance was the MYCOFAST Screening EvolutioN 3 kit.

Results The majority of patients were female (90.7\%), 57.9\% of the patients had $>29$ years of age and $84.3 \%$ did not have a stable union. About $70.0 \%$ of the patients tested positive for UU, $3.0 \%$ for $\mathrm{MH}$, and $27.0 \%$ for both. HIV coinfection was seen in $32.1 \%$ and HPV in $30.0 \%$. Absence of current history of Chlamydia trachomatis was a protective factor for the acquisition of azithromycin resistance $(p=0.04)$. As for the antimicrobials, doxycycline showed sensitivity rates higher than $96 \%$ for both infections, while azithromycin showed $86.8 \%$ of sensitivity for UU, but resistance of $75 \%$ for $\mathrm{MH}$. Ciprofloxacin showed sensitivity rates lower than $15 \%$ for both infections, while less than 35\% were strains sensible to ofloxacin. Erythromycin resistance rates ranged over $65 \%$ for the UU-MH coinfection, while over $90 \%$ of sample was sensible to tetracycline and clarithromycin resistance rates ranged from $7.1 \%$ for UU to $100 \%$ for $\mathrm{MH}$.

Conclusion The use of ciprofloxacin and ofloxacin is highly debatable considering the high rates of total and intermediate resistance. In our population, doxycycline showed high efficiency and is therefore recommended for the treatment of UU and $\mathrm{MH}$ infections. Monitoring antimicrobial resistance is fundamental for the adequacy of the therapeutic recommendations.

\section{P3.84 ESTIMATING THE ANTIBODY PREVALENCE OF HERPES SIMPLEX VIRUS TYPE 1 AMONG SELECT MIDDLE EAST AND NORTH AFRICA POPULATIONS}

'Gheyath Nasrallah, ${ }^{2}$ Soha Dargham, 'Layla Mohammed, '2Laith Abu-Raddad. 'Qatar University, Doha, Qatar; ${ }^{2}$ Weill Cornell Medicine-Qatar, Doha, Qatar

\section{$10.1136 /$ sextrans-2017-053264.319}

Introduction Evidence indicates a growing role for herpes simplex virus type-1 (HSV-1) as a sexually transmitted infection and as the etiological agent of genital herpes, but HSV-1 epidemiology in the Middle East and North Africa (MENA) remains poorly understood. We aimed to measure HSV-1 antibody prevalence among select MENA populations and to characterise the infection's age-distribution.

Methods Evidence indicates a growing role for herpes simplex virus type-1 (HSV-1) as a sexually transmitted infection and as the etiological agent of genital herpes, but HSV-1 epidemiology in the Middle East and North Africa (MENA) remains poorly understood. We aimed to measure HSV-1 antibody prevalence among select MENA populations and to characterise the infection's age-distribution.

Results Country-specific HSV-1 prevalence was estimated for 10 MENA national populations of male blood donors residing in Qatar. HSV-1 prevalence was estimated at 97.5\% $(95 \%$ confidence interval (CI) 95.3\%-98.7\%) among Egyptians, 92.6\% (95\% CI 87.2\%-95.8\%) among Yemenis, 90.7\% (95\% CI 84.5\%-94.6\%) among Sudanese, 88.5\% (95\% CI 83.9\%92.6\%) among Syrians, 86.5\% (95\% CI 81.0\%-90.5\%) among
Jordanians, $82.3 \%$ (95\% CI 78.2\%-85.7\%) among Qataris, $81.4 \%$ (95\% CI 73.3\%-87.5\%) among Iranians, 81.4\% (95\% CI 73.4\%-87.4\%) among Lebanese, $80.5 \%$ (95\% CI $74.2 \%$ $85.2 \%$ ) among Palestinians, and $77.0 \%$ (95\% CI $70.7 \%$ 82.3\%) among Pakistanis. Age-specific HSV-1 prevalence was estimated among male blood donors from Egypt, the Fertile Crescent (Iraq, Jordan, Lebanon, Palestine and Syria) and Qatar. There was a trend of growing HSV-1 prevalence with age among the Fertile Crescent and Qatari nationals. HSV-1 prevalence increased from 70.0\% (95\% CI 56.3\%-80.9\%) for those aged $<24$ years up to $98.0 \%$ (95\% CI $89.5 \%-99.7 \%$ ) for those aged $\geq 55$ years in the Fertile Crescent. Similar pattern was observed for Qatar, but for Egypt, prevalence was steadily above $90 \%$ for all age groups. Our results showed no significant association between sex and HSV-1 seropositivity.

Conclusion HSV-1 prevalence in MENA continues at high level, but for the majority of nationalities, at considerably lower levels than historical levels. The decline in prevalence is most pronounced among youth. As much as a third of those $<30$ years of age are reaching sexual debut with no protective antibodies against HSV-1 genital acquisition, and accordingly, are at risk of genital herpes.

\section{P3.85 EVALUATION OF GENTAMICIN SUSCEPTIBILITY AND RESISTANCE PHENOTYPES OF NEISSERIA GONORRHOEAE ISOLATES IN ARGENTINA}

${ }^{1} \mathrm{R}$ Gianecini, ${ }^{1} \mathrm{C}$ Oviedo, ${ }^{2} \mathrm{GASSP}-\mathrm{AR},{ }^{1}$ Galarza P. ${ }^{1}$ Servicio de Enfermedades de Transmisión Sexual, Instituto Nacional de Enfermedades Infecciosas (INEI)-ANLIS "Dr. Carlos G. Malbrán", Ciudad Autónoma de Buenos Aires, Argentina.; 'Gonococcal Antimicrobial Susceptibility Surveillance Programme-Argentina (GASSP-AR)

\subsection{6/sextrans-2017-053264.320}

Introduction The emergence of multidrug resistance in N. gonorrhoeae highlights the need to consider alternatives for future therapeutic use. Nowadays, the aminoglycoside gentamicin is considered a possible treatment option for gonorrhoea, particularly in dual therapy. Gentamicin has been used successfully for several years in the treatment of gonococcal urethritis in Malawi. However, treatment failures have been reported. In Argentina, N. gonorrhoeae isolates with multiple resistance profiles to different antimicrobials, including cefixime and ceftriaxone, have been detected. Therefore, the knowledge of the susceptibility to new antimicrobial options for gonorrhoea treatment is required. The aim of this study was to determine the susceptibility to gentamicin of $N$. gonorrhoeae isolates with different resistance profiles in 2013 and 2015.

Methods We studied 237 N. gonorrhoeae isolates obtained in 2013 and 2015 from the GASSP-AR. The MICs to penicillin, tetracycline, ciprofloxacin, cefixime, ceftriaxone, azithromycin and gentamicin were determined by the agar dilution test according to CLSI guidelines.

Results Gentamicin MICs ranged from 2 to $16 \mu \mathrm{g} / \mathrm{ml}$. The $\mathrm{MIC}_{50}$ and $\mathrm{MIC}_{90}$ were $8 \mu \mathrm{g} / \mathrm{ml}$. The rate of isolates with MIC $8 \mu \mathrm{g} / \mathrm{ml}$ was $67.1 \%$, while $30.4 \%$ showed a MIC of $4 \mu \mathrm{g} / \mathrm{ml}$. A $75.5 \%$ of the isolates included in this study showed resistance to one or more of the following antibiotics: penicillin (40.5\%), tetracycline (30.4\%), ciprofloxacin $(59.9 \%)$, and $5.5 \%$ of the isolates showed decreased susceptibility to cefixime and ceftriaxone. A total of 15 resistance phenotypes 